\title{
Kolmogoroff's Strong Law of Large Numbers holds for pairwise uncorrelated random variables
}

Original version written on May 8, 2020; Text last updated January 31, 2022; Journal information updated on October 27, 2021

\begin{abstract}
Using the approach of N. Etemadi for the Strong Law of Large Numbers $(S L L N)$ in [1] and its elaboration in [5], I give weaker conditions under which the SLLN still holds, namely for pairwise uncorrelated (and also for "quasi uncorrelated") random variables. I am focusing in particular on random variables which are not identically distributed. My approach here leads to another simple proof of the classical SLLN.

Published in (English edition): Theory of Probability and its Applications, 2021, Volume 66, Issue 2, Pages 263-275.

https://epubs.siam.org/doi/10.1137/S0040585X97T990381.

DoI: https://doi.org/10.1137/S0040585X97T990381.

Published in (Russian edition): Teoriya Veroyatnostei $i$ ee Primeneniya (this is the Russian edition of Theory of Probability and its Applications), 2021, Volume 66, Issue 2, Pages 327-341. (Submitted November 23, 2020; Accepted November 25, 2020).

http://mi.mathnet.ru/tvp5459.

DoI: https://doi.org/10.4213/tvp5459.
\end{abstract}

\section{Introduction and results}

In publication [1] from 1981, N. Etemadi weakened the requirements of Kolmogoroff's first SLLN for identically distributed random variables and provided an elementary proof of a more general SLLN. I will first state the main Theorem of [1] after introducing some notations that I will use.

Notation. Throughout the paper, $(\Omega, \mathcal{A}, \mathrm{P})$ is a probability space. All random variables (usually denoted by $X$ or $X_{n}$ ) are assumed to be measurable functions from $\Omega$ to the set of real numbers $\mathbb{R}$. If $X$ is a random variable, I write $\mathrm{E} X$ for its expected value and $\vee X$ for its variance.

Etemadi's Theorem ([1]). Let $\left(X_{n}\right)_{n \in \mathbb{N}}$ be a sequence of pairwise independent, identically distributed random variables with $\mathrm{E}\left|X_{1}\right|<\infty$. Then

$$
\lim _{n \rightarrow \infty} \frac{X_{1}+X_{2}+\cdots+X_{n}}{n}=\mathrm{E} X_{1} \quad \text { almost surely (a.s.). }
$$

${ }^{*}$ The paper was initially written for a student seminar on probability theory under supervision of Prof. Jean Bertoin, University of Zürich in early 2019. At the time I was 15 years old. My email address is mail@maximilianjanisch.com. Currently I am a graduate student at the Institute of Mathematics, University of Zürich (190, Winterthurerstrasse, 8057 Zürich, Switzerland). 
Remark 1. Following Remark 2 from chapter 4.3 in Shiryaev's book [4], we notice that if $\mathrm{E} X_{1}=\infty$, then (1) still holds.

The main improvement of Etemadi's Theorem compared to Kolmogoroff's first SLLN is the replacement of independence by pairwise independence. The condition "identically distributed" still remains. There have been several extentions of this Theorem. For example, Chandra and Goswami gave a sufficient SLLN condition for non-identically distributed random variables. For convenience, I will from now on always use the notation $S_{n}:=X_{1}+\cdots+X_{n}$.

Theorem 1 (Chandra, Goswami [3]). Let $\left(X_{n}\right)_{n \in \mathbb{N}}$ be a sequence of pairwise independent random variables such that

$$
\int_{0}^{\infty} \sup _{n \in \mathbb{N}} \mathrm{P}\left(\left|X_{n}\right|>t\right) \mathrm{d} t<\infty
$$

Then

$$
\lim _{n \rightarrow \infty} \frac{S_{n}-\mathrm{E} S_{n}}{n}=0 \quad \text { a.s. }
$$

However, Etemadi's arguments enable an elegant proof for large classes of non-identically distributed random variables. First, let me introduce a few useful notions:

Definition 1. A sequence $\left(X_{n}\right)_{n \in \mathbb{N}}$ of (arbitrarily distributed) random variables is said to

i. Satisfy the Kolmogoroff condition if and only if each one of the $X_{n}^{\prime} s, n \in \mathbb{N}$ has finite variance $\mathrm{V} X_{n}$ and

$$
\sum_{n \in \mathbb{N}} \frac{\mathrm{V} X_{n}}{n^{2}}<\infty .
$$

ii. Be quasi uncorrelated if and only if each one of the $X_{n}^{\prime} s, n \in \mathbb{N}$ has finite variance and there exists a positive constant $c$ such that $\mathrm{V} S_{n} \leq c \sum_{k=1}^{n} \mathrm{~V} X_{k}$ for all $n \in \mathbb{N}$.

iii. Satisfy the $S L L N$ if and only if

$$
\lim _{n \rightarrow \infty} \frac{S_{n}-\mathrm{E} S_{n}}{n}=0 \quad \text { a.s. }
$$

Notice that if $a:=\lim _{n \rightarrow \infty} \mathrm{E} X_{n}$ exists, this is (by Cesàro summation) equivalent to the relation $\lim _{n \rightarrow \infty} \frac{1}{n} S_{n}=a$ a.s.

Theorem 2 (SLLN under the Kolmogoroff condition (2)). Let $\left(X_{n}\right)_{n \in \mathbb{N}}$ be a sequence of nonnegative, quasi uncorrelated random variables satisfying the Kolmogoroff condition (2) such that $A:=\sup _{n \in \mathbb{N}} \frac{\mathrm{E} S_{n}}{n}<\infty$. Then the sequence $\left(X_{n}\right)_{n \in \mathbb{N}}$ satisfies the SLLN.

Remark 2. After having finished the proof of this Theorem and the proof of Theorem 3, I became aware of the paper by Korchevsky [2], where the above Theorem 2 is formulated as Lemma 1. Korchevsky refers to the work of Chandra-Goswami [3]. For completeness, I will give a detailed proof of Theorem 2, whereas the proof in [3] is only briefly outlined.

Remark 3. The condition $\sup _{n \in \mathbb{N}} \frac{E S_{n}}{n}<\infty$ can be replaced by $\sup _{n \in \mathbb{N}} \frac{E S_{n}}{b_{n}}<\infty$ for any unbounded, non-decreasing sequence of positive real numbers $b_{n}$. In that case, if the condition $\sum_{n=1}^{\infty} \frac{\mathrm{V} X_{n}}{n^{2}}<\infty$ is replaced by $\sum_{n=1}^{\infty} \frac{\mathrm{V} X_{n}}{b_{n}^{2}}<\infty$, then the sequence $\left(X_{n}\right)_{n \in \mathbb{N}}$ will satisfy $\lim _{n \rightarrow \infty} \frac{S_{n}-\mathrm{E} S_{n}}{b_{n}}=0$ a.s. During the proof I will make remarks that show how the proof has to be adapted in order to work for such $b_{n}$.

Remark 4. Of course Theorem 2 is also true if non-negative is replaced by a.s. non-negative. For my purposes here, these two notions can be seen as synonymous. 
Remark 5. If the $X_{n}$ 's are pairwise uncorrelated, we have the equality $\vee S_{n}=\sum_{k=1}^{n} \vee X_{k}(\mathrm{Bi}$ enaymé) and thus the $X_{n}$ are, in particular, quasi uncorrelated.

Remark 6. If $X$ is a random variable, I use the notation ess inf $X$ for the essential infimum of $X$, i.e. the largest number $r$ such that $X \geq r$ a.s. Applying Theorem 2 to the transformation $\tilde{X}_{n}:=X_{n}-\operatorname{ess} \inf X_{n}$ shows that the condition $\sup _{n \in \mathbb{N}} \frac{E S_{n}}{n}<\infty$ can be replaced by the weaker condition

$$
\sup _{n \in \mathbb{N}} \frac{\mathrm{E} S_{n}-\sum_{k=1}^{n} \operatorname{essinf} X_{k}}{n}<\infty .
$$

Since for any numerical sequence $a_{n} \geq 0, \sup _{n \in \mathbb{N}} a_{n}=\infty$ implies

$$
\limsup _{n \rightarrow \infty} a_{n}=\lim _{n \rightarrow \infty} \sup _{k \geq n} a_{k}=\infty
$$

condition (3) is equivalent to

$$
\limsup _{n \rightarrow \infty} \frac{\mathrm{E} S_{n}-\sum_{k=1}^{n} \operatorname{ess} \inf X_{k}}{n}<\infty .
$$

Remark 7. My proof of Theorem 2 relies heavily on the proof by Csörgo et al. [5]. In their paper, it is also shown that the condition of non-negativity cannot be removed. More precisely, in their Theorem 4, the authors construct a sequence of pairwise uncorrelated random variables $X_{n}$ satisfying the Kolmogoroff condition (2) and the condition

$$
\lim _{n \rightarrow \infty} \frac{\sum_{k=1}^{n} \mathrm{E}\left|X_{k}-\mathrm{E} X_{k}\right|}{n}=0
$$

and

$$
\limsup _{n \rightarrow \infty} \frac{\left|S_{n}-\mathrm{E} S_{n}\right|}{n}=\infty
$$

If we require the $X_{n}$ to be pairwise independent instead of just pairwise uncorrelated, the non-negativity assumption can be dropped:

Corollary 2.1. Let $\left(X_{n}\right)_{n \in \mathbb{N}}$ be a sequence of pairwise independent random variables satisfying the Kolmogoroff condition (2) and suppose further that

$$
\limsup _{n \rightarrow \infty} \frac{\sum_{k=1}^{n} \mathrm{E}\left|X_{k}-\mathrm{E} X_{k}\right|}{n}<\infty .
$$

Then the sequence $\left(X_{n}\right)_{n \in \mathbb{N}}$ satisfies the $S L L N$.

Remark 8. Thanks to linearity of $\mathrm{E}$, the non-negativity condition in Theorem 2 can be replaced by a.s. uniform boundedness of each $X_{n}$ (or even of $X_{n}-\mathrm{E} X_{n}$ ) from below or above. For example, if each $X_{n}$ is bounded from below by the real number $r$, then Theorem 2 can be applied to $\tilde{X}_{n}:=X_{n}-r$. In fact, one can always try to use the transformation $\tilde{X}_{n}:=X_{n}-\operatorname{ess}_{\inf } \operatorname{lN}_{n \in \mathbb{N}} X_{n}$ and check if that transformation satiesfies all conditions of Theorem 2.

Remark 9. Kolmogoroff's classical SLLN is very similar to Corollary 2.1, except that pairwise independence is replaced by independence but the auxilliary condition (4) is dropped. However, as is proven in [5], Theorem 3, there is a sequence of pairwise independent random variables $\left(X_{n}\right)_{n \in \mathbb{N}}$ satisfying the Kolmogoroff condition (2) such that the SLLN is not satisfied. Hence, an auxilliary condition like (4) in Corollary 2.1 is necessary. 
We can try to relax the Kolmogoroff condition (2) for the $X_{n}$ using truncation arguments. For this, however, the condition that all $X_{n}$ 's are pairwise uncorrelated has to be replaced by a condition of pairwise independence. The reason for this is that the truncations of the $X_{n}$ 's are still pairwise independent if the $X_{n}$ 's are pairwise independent, while the truncations need not be pairwise uncorrelated if the $X_{n}$ 's are only pairwise uncorrelated.

More precisely, the truncations of $X_{n}$ that I will look at are $Y_{n}:=X_{n} \cdot 1_{\left\{X_{n} \leq n\right\}}$, where $1_{\left\{X_{n} \leq n\right\}}$ is the indicator function of the event $\left\{X_{n} \leq n\right\}$.

Remark 10. It should be noted that the truncated variables $Y_{n}, n \in \mathbb{N}$, do not have to satisfy the Kolmogoroff condition (2)! It depends on the $X_{n}$ 's. For example, if the $X_{n}$ 's are random variables with $\mathrm{P}\left(X_{n}=n\right)=\mathrm{P}\left(X_{n}=0\right)=\frac{1}{2}$, then $\mathrm{V}\left(Y_{n}\right)=\mathrm{V}\left(X_{n}\right)=\frac{n^{2}}{4}$, which is too large for the Kolmogoroff condition (2) to hold. In this case, however, it is not surprising, because the sequence $\left(X_{n}\right)_{n \in \mathbb{N}}$ also does not satisfy the SLLN. Interestingly, the sequence $\left(X_{n}\right)_{n \in \mathbb{N}}$ does not even satisfy the Weak Law of Large Numbers (WLLN). Indeed, let $\tilde{X}_{n}=X_{n}-\mathrm{E} X_{n}$. Then $S_{n}-\mathrm{E} S_{n}=\sum_{k=1}^{n} \tilde{X}_{k}$ and $\mathrm{P}\left(\tilde{X}_{n}=-\frac{n}{2}\right)=\mathrm{P}\left(\tilde{X}_{n}=\frac{n}{2}\right)=\frac{1}{2}$, so that for $n \geq 2$,

$$
\mathrm{P}\left(S_{n}-\mathrm{E} S_{n} \geq \frac{n}{2}\right)=\mathrm{P}\left(\sum_{k=1}^{n} \tilde{X}_{k} \geq \frac{n}{2}\right)=\frac{\mathrm{P}\left(\sum_{k=1}^{n-1} \tilde{X}_{k} \geq 0\right)+\mathrm{P}\left(\sum_{k=1}^{n-1} \tilde{X}_{k} \geq n\right)}{2} \geq \frac{1}{4}
$$

where the last inequality is true by the symmetry of $\tilde{X}_{k}$, i.e. $\mathrm{P}\left(\sum_{k=1}^{n-1} \tilde{X}_{k} \geq 0\right) \geq \frac{1}{2}$.

Remark 11. Since the SLLN implies the WLLN, the sequence of random variables from Remark 10 satisfy neither the SLLN nor the WLLN. For completeness, I refer to examples 15.3 and 15.4 in [6] for sequences of random variables that satisfy the WLLN but not the SLLN.

Theorem 3 (SLLN for random variables with the Kolmogoroff condition for the truncations). Let $\left(X_{n}\right)_{n \in \mathbb{N}}$ be a sequence of non-negative, pairwise independent random variables such that the truncated sequence $\left(Y_{n}\right)_{n \in \mathbb{N}}$, where $Y_{n}:=X_{n} \cdot 1_{\left\{X_{n} \leq n\right\}}$, satisfies the Kolmogoroff condition (2) as well as (4) and such that $X_{n}-Y_{n}$ goes to 0 in $L^{1}$-sense and a.s. as $n \rightarrow \infty$. Then the sequence $\left(X_{n}\right)_{n \in \mathbb{N}}$ satisfies the SLLN.

Remark 12. Remark 8 also applies to Theorem 3.

Of course, we can slightly modify the assumptions to avoid non-negativity. The following two standard notations, $X_{n}^{+}:=\max \left\{X_{n}, 0\right\}$ and $X_{n}^{-}:=-\min \left\{X_{n}, 0\right\}$, will be used.

Corollary 3.1. Let $\left(X_{n}\right)_{n \in \mathbb{N}}$ be a sequence of random variables such that both sequences $\left(X_{n}^{+}\right)_{n \in \mathbb{N}}$ and $\left(X_{n}^{-}\right)_{n \in \mathbb{N}}$ satisfy the conditions of Theorem 3. Then the sequence $\left(X_{n}\right)_{n \in \mathbb{N}}$ satisfies the $S L L N$.

Finally, I want to prove a (well-known) statement which shows that Etemadi's result follows from Corollary 3.1 since identically distributed random variables "behave nicely":

Theorem 4. Let $\left(X_{n}\right)_{n \in \mathbb{N}}$ be a sequence of non-negative, identically distributed, integrable random variables. Then the truncated sequence $\left(Y_{n}\right)_{n \in \mathbb{N}}$, defined by $Y_{n}:=X_{n} \cdot 1_{\left\{X_{n} \leq n\right\}}$, satisfies the Kolmogoroff condition, and, as $n \rightarrow \infty, X_{n}-Y_{n} \rightarrow 0$ both in $L^{1}$-sense and a.s.

If we are given any identically distributed, pairwise independent random variables $X_{1}, X_{2}, \ldots$ with $\mathrm{E}\left|X_{1}\right|<\infty$, we can apply Theorem 4 to each one of the sequences $\left(X_{n}^{+}\right)_{n \in \mathbb{N}}$ and $\left(X_{n}^{-}\right)_{n \in \mathbb{N}}$ to see that all conditions of Corollary 3.1 are satisfied. As a consequence, we obtain the conclusion of Etemadi's Theorem. 


\section{Examples}

In order to illustrate my results I will now give two examples.

Example 1. Let $X$ be a (continuous) uniformly distributed random variable over the interval $[-1,1]$. Define the sequence

$$
X_{n}:=\cos (2 \pi n X) \text { for } n \in \mathbb{N} .
$$

Claim. The following three statements are true:

1) The sequence $\left(X_{n}\right)_{n \in \mathbb{N}}$ is pairwise uncorrelated.

2) Each pair $\left(X_{i}, X_{j}\right)$ for $i, j \in \mathbb{N}$ is not independent.

3) The sequence $\left(X_{n}\right)_{n \in \mathbb{N}}$ satisfies the $S L L N$.

Remark 13. As will be seen in the proof, my Theorem 2 can be used to prove that $\left(X_{n}\right)_{n \in \mathbb{N}}$ satisfies the SLLN. Notice that, because of statement 2), the original Theorem in [5] cannot be applied.

Proof of the statements. Let me start with the most important statement, 3). Consider $\tilde{X}_{n}:=$ $1+X_{n}$. Then (see $\left.(5)\right), \mathrm{E}\left(\tilde{X}_{n}\right)=1+\mathrm{E}\left(X_{n}\right)=1$. Hence $\sup _{n \in \mathbb{N}} \frac{\mathrm{E}\left(\tilde{X}_{1}+\cdots+\tilde{X}_{n}\right)}{n}=1<\infty$. Additionally, all of the random variables $\tilde{X}_{n}$ are in the interval $[0,2]$, which implies that the Kolmogoroff condition is satisfied. Statement 1) guarantees that Theorem 2 can be applied to conclude that the sequence $\left(X_{n}\right)_{n \in \mathbb{N}}$ satisfies the SLLN.

To prove statement 1 ), we need to show that $\mathrm{E}\left(X_{i} X_{j}\right)=\mathrm{E}\left(X_{i}\right) \mathrm{E}\left(X_{j}\right)$ for all $i \neq j$. This is true by the following two simple computations:

$$
\begin{aligned}
\mathrm{E}\left(X_{i}\right)=\int_{-1}^{1} \cos (2 \pi i x) \mathrm{d} x & =0=\mathrm{E}\left(X_{j}\right), \\
\mathrm{E}\left(X_{i} X_{j}\right)=\int_{-1}^{1} \cos (2 \pi i x) \cos (2 \pi j x) \mathrm{d} x & =0 .
\end{aligned}
$$

The last equality follows from the elementary trigonometric identity

$$
\cos (2 \pi i x) \cos (2 \pi j x)=\frac{\cos (2 \pi(i-j) x)+\cos (2 \pi(i+j) x)}{2} .
$$

To prove statement 2), let $\varepsilon>0$ and suppose that $X_{i}>1-\varepsilon$ for some $i \in \mathbb{N}$. This means that $\cos (2 \pi i X)>1-\varepsilon$. Consider the arccos "branch", defined by

$$
\begin{aligned}
\arccos _{i}:[-1,1] & \rightarrow[2 i \pi,(2 i+1) \pi], \\
\cos (x) & \mapsto x
\end{aligned}
$$

This function is continuous and thus there exist $\delta(\varepsilon)>0$ such that

$$
2 \pi i X \in] 2 \pi k-\delta(\varepsilon), 2 \pi k+\delta(\varepsilon)[
$$

for some $k \in \mathbb{Z}$ and such that $\lim _{\varepsilon \rightarrow 0} \delta(\varepsilon)=0$. This means that

$$
\left.X \in \bigcup_{k=-i}^{i}\right] \frac{k}{i}-\frac{\delta(\varepsilon)}{i}, \frac{k}{i}+\frac{\delta(\varepsilon)}{i}[\stackrel{\text { Def. }}{=} D .
$$


Each interval in the union has length $\frac{2 \delta(\varepsilon)}{i}$ and there are $2 i$ intervals. Hence, since cos is 1-Lipschitz, for any $j \in \mathbb{N}, \cos (2 \pi j D)$ has Lebesgue measure at most $4 \delta(\varepsilon)$. If $\varepsilon$ is small enough, we have $4 \delta(\varepsilon)<2=\operatorname{length}([-1,1])$. Hence in that case, as $\mathrm{P}\left(X_{j} \in[a, b]\right)>0$ for any $0 \leq a<b \leq 1$

$$
\mathrm{P}\left(X_{j} \in[-1,1] \backslash \cos (2 \pi j D) \mid X_{i}>1-\varepsilon\right)=0<\mathrm{P}\left(X_{j} \in[-1,1] \backslash \cos (2 \pi j D)\right)
$$

and thus the pair $\left(X_{i}, X_{j}\right)$ is not independent.

Example 2. Let $Z_{n}, n \in \mathbb{N}$, be independent standard normal random variables (i.e. each $Z_{n}$ has a normal distribution with mean 0 and variance 1$)$. Let $W$ be a random variable, independent of every $Z_{n}$ for $n \in \mathbb{N}$, such that $\mathrm{P}(W=0)=\mathrm{P}(W=1)=1 / 2$. Then the statements 1$\left.\left.), 2\right), 3\right)$ from Example 1 also hold for the random variables $X_{n}:=W Z_{n}, n \in \mathbb{N}$.

Proof. To prove 1), note that, by symmetry of each $Z_{n}$ and by the independence assumptions, for all $i \neq j \in \mathbb{N}$,

$$
\mathrm{E}\left(X_{i}\right) \mathrm{E}\left(X_{j}\right)=\mathrm{E}(W) \mathrm{E}\left(Z_{i}\right) \mathrm{E}\left(X_{j}\right)=0 \quad \text { and } \mathrm{E}\left(X_{i} X_{j}\right)=\mathrm{E}\left(W^{2}\right) \mathrm{E}\left(Z_{i}\right) \mathrm{E}\left(Z_{j}\right)=0 .
$$

To prove 2), notice that if there exists any $i \in \mathbb{N}$ for which $X_{i}>0$, then $W=1$. Hence, for all $i, j \in \mathbb{N}$ with $i \neq j$,

$$
\mathrm{P}\left(X_{j}>0 \mid X_{i}>0\right)=\mathrm{P}\left(Z_{j}>0\right)=\frac{1}{2}>\mathrm{P}\left(Z_{j}>0 \text { and } W=1\right)=\frac{1}{4}=\mathrm{P}\left(X_{j}>0\right) .
$$

Finally I will prove 3). Since $\frac{X_{1}+\cdots+X_{n}}{n}=W \frac{Z_{1}+\cdots+Z_{n}}{n}$, and $\frac{Z_{1}+\cdots+Z_{n}}{n}$ has a normal distribution with mean 0 and variance $\frac{1}{n}$, we see that the SLLN holds for the sequence $\left(X_{n}\right)_{n \in \mathbb{N}}$ in question.

Remark 14. Example 2 also is an example of a sequence of random variables for which the SLLN holds, but for which the non-negativity/bounded-from-below assumption in Theorem 2 is not satisfied. If we try to treat this problem by applying Theorem 2 separately to $X_{n}^{+}:=\max \left\{X_{n}, 0\right\}$ and $X_{n}^{-}:=-\min \left\{X_{n}, 0\right\}, n \in \mathbb{N}$, then we will run into other issues because

$$
\mathrm{V}\left(X_{1}^{+}+\cdots+X_{n}^{+}\right)=n \mathrm{~V}\left(X_{1}^{+}\right)+\left(\begin{array}{l}
n \\
2
\end{array}\right)\left(\mathrm{E}\left(X_{1}^{+} X_{2}^{+}\right)-\mathrm{E}\left(X_{1}^{+}\right) \mathrm{E}\left(X_{2}^{+}\right)\right)
$$

while

We can compute ${ }^{1}$

$$
\mathrm{V}\left(X_{1}^{+}\right)+\cdots+\mathrm{V}\left(X_{n}^{+}\right)=n \mathrm{~V}\left(X_{1}^{+}\right)
$$

$$
\mathrm{E}\left(X_{2}^{+}\right)=\mathrm{E}\left(X_{1}^{+}\right)=\int_{0}^{\infty} \mathrm{P}\left(X_{1}^{+}>t\right) \mathrm{d} t=\frac{1}{2} \int_{0}^{\infty} \mathrm{P}\left(Z_{1}>t\right) \mathrm{d} t=\frac{1}{2 \sqrt{2 \pi}} \approx 0.20
$$

and

$$
\mathrm{E}\left(X_{1}^{+} X_{2}^{+}\right)=\int_{0}^{\infty} \mathrm{P}\left(X_{1}^{+} X_{2}^{+}>t\right) \mathrm{d} t=\int_{0}^{\infty} \int_{0}^{\infty} \int_{t / x}^{\infty} \frac{\exp \left(-\frac{x^{2}}{2}\right)}{\sqrt{2 \pi}} \frac{\exp \left(-\frac{y^{2}}{2}\right)}{\sqrt{2 \pi}} \mathrm{d} y \mathrm{~d} x \mathrm{~d} t \approx 0.16 .
$$

In particular, we see that $\mathrm{E}\left(X_{1}^{+} X_{2}^{+}\right)>\mathrm{E}\left(X_{1}^{+}\right) \mathrm{E}\left(X_{2}^{+}\right)$, and hence by $(8), \mathrm{V}\left(X_{1}^{+}+\cdots+X_{n}^{+}\right)$is bigger than some positive constant (independent of $n$ ) times $\left(\begin{array}{l}n \\ 2\end{array}\right)$ for all $n \in \mathbb{N}$. Comparing this with (9) shows that the variables $X_{n}^{+}, n \in \mathbb{N}$ are not even quasi-uncorrelated.

\footnotetext{
${ }^{1}$ See for instance https://mathworld.wolfram.com/Erfc.html for more on the integral of the complementary error function (which I used in the computation of $\int_{0}^{\infty} \mathrm{P}\left(Z_{1}>t\right) \mathrm{d} t$ ). In the second computation, I use that for $t>0$,

$$
\mathrm{P}\left(X_{1}^{+} X_{2}^{+}>t\right)=\mathrm{P}\left(X_{1} X_{2}>t \text { and } X_{1}>0 \text { and } X_{2}>0\right)=\frac{1}{2} \mathrm{P}\left(Z_{1} Z_{2}>t \text { and } Z_{1}>0 \text { and } Z_{2}>0\right) .
$$
}


Remark 15. These two examples were added thanks to a suggestion by Jordan Stoyanov to provide examples of (infinite) sequences of random variables that are pairwise uncorrelated but not pairwise independent. The above examples can be considered as an extension to infinite sequences of a property for a pair of random variables. (Examples of such pairs can be found in [6], chapter 7).

\section{Proofs}

\subsection{Tools for Theorem 2}

Definition 2. From now on, fix real numbers $\alpha>1$ and $\varepsilon>0$. The random variables $X_{n}$ are as in Theorem 2. Recall that, by assumption, $A:=\sup _{n \in \mathbb{N}} \frac{\mathrm{E} S_{n}}{n}<\infty$. Hence it is possible to define the integer $L:=\left\lfloor\frac{A}{\varepsilon}\right\rfloor$, where $\lfloor\bullet\rfloor$ denotes "the largest integer smaller than, or equal to, -" (also known as the "floor part").

For every $n \in \mathbb{N}$ let $m(n):=\left\lfloor\log _{\alpha} n\right\rfloor$. Then

$$
m(n) \rightarrow \infty \text { as } n \rightarrow \infty \text { and } \alpha^{m(n)} \leq n<\alpha^{m(n)+1} \text { for all } n
$$

For a non-negative random variable $X_{n}$, let $s(n) \in\{0, \ldots, L\}$ be natural numbers such that

$$
\frac{\mathrm{E}\left(S_{n}\right)}{n} \in[\varepsilon \cdot s(n), \varepsilon \cdot(s(n)+1)[.
$$

The numbers $s(n)$ are always uniquely well-defined because the intervals in (10) are disjoint and their union contains the interval $[0, A]$.

Definition 3. Let the random variables $X_{n}, n \in \mathbb{N}$, be as in Theorem 2. For any $n \in \mathbb{N}$ and $s \in\{0, \ldots, L\}$, consider the sets

$$
T_{n, s}:=\left\{k \in \mathbb{N}: \alpha^{n} \leq k<\alpha^{n+1} \text { and } \varepsilon \cdot s \leq \frac{\mathrm{E}\left(S_{k}\right)}{k}<\varepsilon \cdot(s+1)\right\} .
$$

For some $n$ and $s, T_{n, s}$ can be empty. However, $T_{m(n), s(n)}$ is never empty as $n \in T_{m(n), s(n)}$ for any $n \in \mathbb{N}$. If $T_{n, s}$ is not empty, set $k(n, s)^{+}:=\max T_{n, s}$ and $k(n, s)^{-}:=\min T_{n, s}$. Otherwise, if $T_{n, s}$ is empty, take

$$
k(n, s)^{+}=k(n, s)^{-}:=\left\lfloor\alpha^{n}\right\rfloor .
$$

From now on, I will use the symbol $k(n, s)^{ \pm}$in statements that are true for both $k(n, s)^{+}$and $k(n, s)^{-}$, respectively. For example, we have $k(n, s)^{ \pm} \geq\left\lfloor\alpha^{n}\right\rfloor \rightarrow \infty$ as $n \rightarrow \infty$.

Remark 16. If we are dealing with $\frac{S_{n}-\mathrm{E} S_{n}}{b_{n}}$ instead of $\frac{S_{n}-\mathrm{E} S_{n}}{n}$ as described in Remark 3, then one has to replace $\frac{\mathrm{E} S_{n}}{n}$ in (10) and (11) with $\frac{\mathrm{E} S_{n}}{b_{n}}$.

The next statement is about the Kolmogoroff condition for the $S_{k(n, s) \pm}$ subsequences.

Lemma 1. Let $\left(X_{n}\right)_{n \in \mathbb{N}}$ be a sequence of random variables satisfying the assumptions of Theorem 1. Then the $S_{k(n, s)^{ \pm}}$subsequences satisfy the Kolmogoroff condition, i.e.

$$
\sum_{n=1}^{\infty} \frac{\mathrm{V}\left(S_{k(n, s)^{ \pm}}\right)}{\left(k(n, s)^{ \pm}\right)^{2}}<\infty \text { for any } s \in\{0, \ldots, L\}
$$

Remark 17. Lemma 1 is not surprising because $\left(X_{n}\right)_{n \in \mathbb{N}}$ satisfies the Kolmogoroff condition (2) and the numbers $k(n, s)^{ \pm}$grow exponentially in $n$. 
Remark 18. If we consider the case of Remark 3, then in the same way one can prove that

$$
\sum_{n=1}^{\infty} \frac{\mathrm{V} S_{k(n, s)^{ \pm}}}{b_{k(n, s)^{ \pm}}^{2}}<\infty
$$

Proof of Lemma 1. Because the variables $X_{n}$ are quasi-uncorrelated, we have

$$
\begin{aligned}
\sum_{n=1}^{\infty} \frac{\mathrm{V} S_{k(n, s)^{ \pm}}}{\left(k(n, s)^{ \pm}\right)^{2}} & \leq c \sum_{n=1}^{\infty}\left(\frac{1}{\left(k(n, s)^{ \pm}\right)^{2}} \sum_{j=1}^{k(n, s)^{ \pm}} \mathrm{V} X_{j}\right) \\
& =c \sum_{j=1}^{\infty}\left(\mathrm{V} X_{j} \sum_{\left\{n: k(n, s)^{ \pm} \geq j\right\}} \frac{1}{\left(k(n, s)^{ \pm}\right)^{2}}\right)
\end{aligned}
$$

(the last equality holds by rearranging the terms). For brevity, I define $\kappa_{j}:=\sum_{\left\{n: k(n, s)^{ \pm} \geq j\right\}} \frac{1}{\left(k(n, s)^{ \pm}\right)^{2}}$. By Definition 3, we have that for all $n, s$,

$$
\left\lfloor\alpha^{n}\right\rfloor \leq k(n, s)^{ \pm} \leq \alpha^{n+1} \text { so that }\left\{n: k(n, s)^{ \pm} \geq j\right\} \subset\left\{n: \alpha^{n} \geq \frac{j}{\alpha}\right\}
$$

It follows that

$$
\kappa_{j} \leq \sum_{\left\{n: \alpha^{n} \geq \frac{j}{\alpha}\right\}} \frac{1}{\left\lfloor\alpha^{n}\right\rfloor}=\frac{\alpha^{2}}{j^{2}} \sum_{n=0}^{\infty} \frac{1}{\left\lfloor\alpha^{n}\right\rfloor^{2}} \leq \frac{\alpha^{2}}{j^{2}} C \frac{\alpha^{2}}{\alpha^{2}-1} \text { for some } C>0 .
$$

In the last inequality, I have used that $\sum_{n=0}^{\infty} \frac{1}{\left\lfloor\left.\alpha^{n}\right|^{2}\right.}$ behaves like a geometric series. Using (14) in (13) we get, as the $X_{n}$ satisfy the Kolmogoroff condition,

$$
\sum_{n=1}^{\infty} \frac{\mathrm{V} S_{k(n, s)^{ \pm}}}{\left(k(n, s)^{ \pm}\right)^{2}} \leq c \cdot C \frac{\alpha^{4}}{\alpha^{2}-1} \sum_{j=1}^{\infty} \frac{\mathrm{V} X_{j}}{j^{2}}<\infty .
$$

This completes the proof of (12).

Lemma 2 (SLLN for the $S_{k(n, s)^{ \pm}}$subsequences). Let $\left(X_{n}\right)_{n \in \mathbb{N}}$ be a sequence of random variables as in Theorem 1. Then the SLLN holds for the subsequences $S_{k(n, s)^{ \pm}}, n \in \mathbb{N}$, i.e. we have

$$
\lim _{n \rightarrow \infty} \frac{S_{k(n, s)^{ \pm}}-\mathrm{E} S_{k(n, s)^{ \pm}}}{k(n, s)^{ \pm}}=0 \text { a.s. for any } s \in\{0, \ldots, L\} .
$$

Remark 19. If we deal with the case of Remark 3, then one can prove in the same way as below that

$$
\lim _{n \rightarrow \infty} \frac{S_{k(n, s)^{ \pm}}-\mathrm{E} S_{k(n, s)^{ \pm}}}{b_{k(n, s)^{ \pm}}}=0 \text { a.s. for all } s \in\{0, \ldots, L\} .
$$

Proof of Lemma 2. Fix $s \in\{0, \ldots, L\}$. For $\delta>0$, define the events

$$
A_{\delta}(n):=\left\{\left|\frac{S_{k(n, s)^{ \pm}}}{k(n, s)^{ \pm}}-\mathrm{E} \frac{S_{k(n, s) \pm}}{k(n, s)^{ \pm}}\right|>\delta\right\} .
$$

Then

$$
\begin{aligned}
A_{\delta}(n) & =\left\{\left|S_{k(n, s)^{ \pm}}-\mathrm{E} S_{k(n, s)^{ \pm}}\right|>k(n, s)^{ \pm} \cdot \delta\right\} \\
& =\left\{\left|S_{k(n, s)^{ \pm}}-\mathrm{E} S_{k(n, s)^{ \pm}}\right|^{2}>\left(k(n, s)^{ \pm}\right)^{2} \cdot \delta^{2}\right\} .
\end{aligned}
$$


We can now use the Chebyshev inequality and Lemma 1 to derive that

$$
\sum_{n=1}^{\infty} \mathrm{P}\left(A_{\delta}(n)\right) \stackrel{\text { Chebyshev }}{\leq} \frac{1}{\delta^{2}} \sum_{n=1}^{\infty} \frac{\mathrm{V} S_{k(n, s)^{ \pm}}}{\left(k(n, s)^{ \pm}\right)^{2}} \stackrel{\text { Lemma } 1}{<} \infty
$$

Consider the sets

$$
A_{\delta}:=\limsup _{n \rightarrow \infty} A_{\delta}(n) .
$$

The relation (16) allows us to apply the Borel-Cantelli Lemma to obtain that $\mathrm{P}\left(A_{\delta}\right)=0$ for any $\delta>0$. This implies the a.s. convergence of $\left|\frac{S_{k(n, s)} \pm}{k(n, s)^{ \pm}}-\mathrm{E} \frac{S_{k(n, s)}}{k(n, s)^{ \pm}}\right|$to 0 as $n \rightarrow \infty$ by standard results from probability theory and thus also proves Lemma 2.

\subsection{Proof of Theorem 2}

Proof of Theorem 2. From now on, I will abbreviate $k(m(n), s(n))^{ \pm}$by $k^{ \pm}$. The idea of the proof is to use the fact that the SLLN holds for the $k^{ \pm}$-subsequence and conclude that it also holds for the sequence $\left(X_{n}\right)_{n \in \mathbb{N}}$ using monotonicity arguments. This is the place where the non-negativity of the $X_{n}$ 's is necessary.

Using definitions 2, 3 and the bound (10), we obtain that for any $\varepsilon>0$ and $n \in \mathbb{N}$ :

$$
k^{-} \leq n \leq k^{+} \quad \text { and } \quad\left|\frac{\mathrm{E} S_{k^{ \pm}}}{k^{ \pm}}-\frac{\mathrm{E} S_{n}}{n}\right| \leq \varepsilon .
$$

Since all $X_{n}$ 's are non-negative, we have

$$
0 \leq S_{n} \leq S_{n^{\prime}} \text { for all } n \leq n^{\prime} \text {. In particular, } 0 \leq \mathrm{E} S_{n} \leq \mathrm{E} S_{n^{\prime}} .
$$

Note that by Definitions 2 and 3, we have $n \geq k^{+} / \alpha$. Thus $1 / n \leq \alpha / k^{+}$and

$$
\left(\frac{1}{n}-\frac{\alpha}{k^{+}}\right) S_{k^{+}}+(\alpha-1) \frac{\mathrm{E} S_{k^{+}}}{k^{+}} \leq(\alpha-1) B,
$$

since $\frac{\mathrm{E} S_{k^{+}}}{k^{+}} \leq B$ and $\left(\frac{1}{n}-\frac{\alpha}{k^{+}}\right) S_{k^{+}} \leq 0$. Bound (19) is equivalent to

$$
\frac{S_{k^{+}}}{n}-\frac{\mathrm{E} S_{k^{+}}}{k^{+}} \leq \frac{\alpha}{k^{+}}\left(S_{k^{+}}-\mathrm{E} S_{k^{+}}\right)+(\alpha-1) B \text {. }
$$

It is also true that $\alpha k^{-} \geq n$. Thus $1 /\left(\alpha k^{-}\right) \leq 1 / n$. By the definition of $B$,

$$
\begin{aligned}
-\left(1-\frac{1}{\alpha}\right) B+\frac{1}{\alpha k^{-}}\left(S_{k^{-}}-\mathrm{E} S_{k^{-}}\right) & \leq-\left(1-\frac{1}{\alpha}\right) \frac{1}{k^{-}} \mathrm{E} S_{k^{-}}+\frac{1}{\alpha k^{-}}\left(S_{k^{-}}-\mathrm{E} S_{k^{-}}\right) \\
& =\frac{S_{k^{-}}}{\alpha k^{-}}-\mathrm{E} \frac{S_{k^{-}}}{k^{-}} \leq \frac{S_{k^{-}}}{n}-\mathrm{E} \frac{S_{n}}{n}+\varepsilon
\end{aligned}
$$

where $\frac{S_{k^{-}}}{\alpha k^{-}} \leq \frac{S_{k^{-}}}{n}$ was used in the last inequality. In conclusion, referring to (21), (18) and (20),

$$
\begin{aligned}
-\varepsilon-\left(1-\frac{1}{\alpha}\right) B+\frac{1}{\alpha k^{-}}\left(S_{k^{-}}-\mathrm{E} S_{k^{-}}\right) & \leq \frac{S_{k^{-}}}{n}-\frac{\mathrm{E} S_{n}}{n} \\
& \leq \frac{1}{n}\left(S_{n}-\mathrm{E} S_{n}\right) \\
& \leq \frac{1}{n} S_{k^{+}}-\frac{1}{k^{+}} \mathrm{E} S_{k^{+}}+\varepsilon \\
& \leq \frac{\alpha}{k^{+}}\left(S_{k^{+}}-\mathrm{E} S_{k^{+}}\right)+(\alpha-1) B+\varepsilon
\end{aligned}
$$


Lemma 2 guarantees that for any $\alpha>1$ and $\varepsilon>0$, there exists a measurable event $\Omega_{\alpha, \varepsilon} \subset \Omega$ such that

$$
\mathrm{P}\left(\Omega_{\alpha, \varepsilon}\right)=1 \quad \text { and } \quad \lim _{n \rightarrow \infty} \frac{S_{k^{ \pm}}(\omega)-\mathrm{E} S_{k^{ \pm}}}{\alpha k^{ \pm}}=0 \text { for all } \omega \in \Omega_{\alpha, \varepsilon} .
$$

Using the previously proven inequality (22) yields

$$
-\varepsilon-\left(1-\frac{1}{\alpha}\right) B \leq \liminf _{n \rightarrow \infty} \frac{1}{n}\left(S_{n}(\omega)-\mathrm{E} S_{n}\right) \leq \limsup _{n \rightarrow \infty} \frac{1}{n}\left(S_{n}(\omega)-\mathrm{E} S_{n}\right) \leq(\alpha-1) B+\varepsilon
$$

for every $\omega \in \Omega_{\alpha, \varepsilon}$. This holds for any $\alpha>1$ and $\varepsilon>0$. By $\sigma$-additivity of $\mathrm{P}$, we have

$$
\mathrm{P}\left(\bigcap_{k, m \in \mathbb{N}} \Omega_{1+\frac{1}{m}, \frac{1}{k}}\right)=1 .
$$

If we substitute $\alpha=1+\frac{1}{m}$ and $\varepsilon=\frac{1}{k}$ for $m, k \in \mathbb{N}$ into (23) and let $m$ and $k$ go to $\infty$ simultaneously, then both "outer sides" of (23) go to 0. It follows from (24) that

$$
\lim _{n \rightarrow \infty} \frac{1}{n}\left(S_{n}(\omega)-\mathrm{E} S_{n}\right)=0
$$

for almost every $\omega \in \Omega$.

This completes the proof of Theorem 2 .

Remark 20. Note that (23) becomes

$$
-\varepsilon-\left(1-\frac{1}{\alpha}\right) B \leq \liminf _{n \rightarrow \infty} \frac{1}{b_{n}}\left(S_{n}(\omega)-\mathrm{E} S_{n}\right) \leq \limsup _{n \rightarrow \infty} \frac{1}{b_{n}}\left(S_{n}(\omega)-\mathrm{E} S_{n}\right) \leq(\alpha-1) B+\varepsilon
$$

in the case described in Remark 3.

\subsection{Proofs of Corollary 2.1 and Corollary 3.1}

Proof of Corollary 2.1. We can assume without loss of generality that the variables $X_{n}, n \in \mathbb{N}$ are centered, i.e., that $\mathrm{E} X_{n}=0$ for all $n \in \mathbb{N}$ (otherwise consider $\tilde{X}_{n}:=X_{n}-\mathrm{E} X_{n}$ ). Since the variables $X_{n}, n \in \mathbb{N}$ are pairwise independent, we see that the sequences $\left(X_{n}^{+}\right)_{n \in \mathbb{N}}$ and $\left(X_{n}^{-}\right)_{n \in \mathbb{N}}$, consist of non-negative and pairwise independent random variables and that each sequences satisfies the Kolmogoroff condition (since $\mathrm{V} X_{n}^{+} \leq \mathrm{V} X_{n}$ and $\mathrm{V} X_{n}^{-} \leq \mathrm{V} X_{n}$ ), ${ }^{2}$ and that

$$
\sup _{n \in \mathbb{N}} \frac{\sum_{k=1}^{n} \mathrm{E} X_{k}^{+}}{n} \leq \sup _{n \in \mathbb{N}} \frac{\sum_{k=1}^{n} \mathrm{E}\left|X_{k}\right|}{n}<\infty .
$$

The analogous relation is true for $\left(X_{k}^{-}\right)_{k \in \mathbb{N}}$.

Hence, by Theorem 2, we get

$$
\begin{aligned}
& \lim _{n \rightarrow \infty} \frac{\sum_{k=1}^{n} X_{k}^{+}-\mathrm{E} X_{k}^{+}}{n}=0, \\
& \lim _{n \rightarrow \infty} \frac{\sum_{k=1}^{n} X_{k}^{-}-\mathrm{E} X_{k}^{-}}{n}=0 .
\end{aligned}
$$

Adding the two proves the Corollary.

Proof of Corollary 3.1. By using the same decomposition as before and then directly applying Theorem 3 to $\left(X_{n}^{+}\right)_{n \in \mathbb{N}}$ and $\left(X_{n}^{-}\right)_{n \in \mathbb{N}}$, we get $(25 \mathrm{a})$ and $(25 \mathrm{~b})$, so that we can conclude once again by adding.

\footnotetext{
${ }^{2}$ For any $n \in \mathbb{N}$, one has $\mathrm{V}\left(X_{n}\right)=\mathrm{E}\left(X_{n}^{2}\right)-\mathrm{E}\left(X_{n}\right)^{2}=\mathrm{E}\left(X_{n}^{2}\right) \geq \mathrm{E}\left(\left(X_{n}^{+}\right)^{2}\right) \geq \mathrm{V}\left(X_{n}^{+}\right)$. The same is true for $X_{n}^{-}$. In the first inequality, I have used that $\left(X_{n}^{+}\right)^{2}=\max \left\{X_{n}, 0\right\}^{2} \leq X_{n}^{2}$.
} 


\subsection{Tools for Theorem 3}

I start with a statement showing that the expected value of the truncations does not differ too much from the original.

Lemma 3. Let $\left(X_{n}\right)_{n \in \mathbb{N}}$ be a sequence of non-negative random variables such that, as $n \rightarrow \infty$, $X_{n}-Y_{n} \rightarrow 0$ in $L^{1}$-sense, where $Y_{n}=X_{n} \cdot 1_{\left\{X_{n} \leq n\right\}}$. Then, for $T_{n}=Y_{1}+\cdots+Y_{n}$,

$$
\lim _{n \rightarrow \infty} \frac{\mathrm{E} S_{n}-\mathrm{E} T_{n}}{n}=0
$$

Proof. The proof is straight-forward. Indeed,

$$
0 \leq \lim _{n \rightarrow \infty} \frac{\mathrm{E} S_{n}-\mathrm{E} T_{n}}{n}=\lim _{n \rightarrow \infty} \frac{\sum_{k=1}^{n} \mathrm{E}\left(X_{k}-Y_{k}\right)}{n}=0,
$$

where the last equality is true by Cesàro summation.

The next result shows that if the SLLN holds for $\left(T_{n}\right)_{n \in \mathbb{N}}$, then it also holds for $\left(S_{n}\right)_{n \in \mathbb{N}}$.

Lemma 4. Let $\left(X_{n}\right)_{n \in \mathbb{N}}$ be a sequence of non-negative random variables such that, as $n \rightarrow \infty$, $X_{n}-Y_{n} \stackrel{\text { a.s. }}{\longrightarrow} 0$. Using the same notation as in Lemma 3, we have

$$
\lim _{n \rightarrow \infty} \frac{S_{n}-T_{n}}{n}=0 \text { a.s. }
$$

Proof. Same idea as in Lemma 3,

$$
0 \leq \frac{S_{n}-T_{n}}{n}=\frac{\sum_{k=1}^{n} X_{k}-Y_{k}}{n} \stackrel{\text { a.s. }}{\longrightarrow} 0 \text { as } n \rightarrow \infty
$$

by Cesàro-summation.

\subsection{Proof of Theorem 3}

Proof of Theorem 3. From Lemmas 3 and 4, we know that for almost every $\omega \in \Omega$, we have

$$
\begin{aligned}
\lim _{n \rightarrow \infty} \frac{S_{n}(\omega)-\mathrm{E} S_{n}}{n} & =\lim _{n \rightarrow \infty} \frac{T_{n}(\omega)-\mathrm{E} T_{n}}{n}+\frac{\mathrm{E} T_{n}-\mathrm{E} S_{n}}{n}+\frac{S_{n}(\omega)-T_{n}(\omega)}{n} \\
& =\lim _{n \rightarrow \infty} \frac{T_{n}(\omega)-\mathrm{E} T_{n}}{n} .
\end{aligned}
$$

Since the sequence $\left(Y_{n}\right)_{n \in \mathbb{N}}$ satisfies the Kolmogoroff condition by assumption and since the variables $Y_{n}, n \in \mathbb{N}$ are pairwise independent, we can apply Theorem 2 to obtain that

$$
\lim _{n \rightarrow \infty} \frac{T_{n}-\mathrm{E} T_{n}}{n}=0 \text { a.s. }
$$

This completes the proof. 


\subsection{Proof of Theorem 4}

Proof of Theorem 4. I will first prove that the sequence $\left(Y_{n}\right)_{n \in \mathbb{N}}$ satisfies the Kolmogoroff condition. For this, let us bound the tail sums of the convergent series $\sum_{j=1}^{\infty} \frac{1}{j^{2}}$. Recall that $\sum_{j=1}^{\infty} \frac{1}{j^{2}}=\frac{\pi^{2}}{6}$, which is the famous Basel problem. I claim that

$$
\sum_{j=k}^{\infty} \frac{1}{j^{2}} \leq \frac{1}{k} \cdot \frac{\pi^{2}}{6} \text { for any } k \in \mathbb{N} .
$$

Notice that if $k=1$, then (27) is true (we have equality). For $k \geq 2$, we have

$$
\sum_{j=k}^{\infty} \frac{1}{j^{2}}<\int_{k-1}^{\infty} \frac{1}{x^{2}} \mathrm{~d} x=\frac{1}{k-1} \leq \frac{2}{k}<\frac{1}{k} \cdot \frac{\pi^{2}}{6} .
$$

Now, using (27) and the abbreviation $g_{k}:=1_{\left\{k<X_{1} \leq k+1\right\}}$,

$$
\begin{aligned}
\sum_{j=1}^{\infty} \frac{\mathrm{V} Y_{j}}{j^{2}} & \leq \sum_{j=1}^{\infty} \frac{\mathrm{E}\left(Y_{j}^{2}\right)}{j^{2}}=\sum_{j=1}^{\infty} \frac{\mathrm{E}\left(X_{1}^{2} \cdot 1_{\left\{X_{1} \leq j\right\}}\right)}{j^{2}} \\
& =\lim _{N \rightarrow \infty} \sum_{j=1}^{N}\left(\frac{1}{j^{2}} \sum_{k=0}^{j-1} \mathrm{E}\left(X_{1}^{2} \cdot g_{k}\right)\right) \\
& =\lim _{N \rightarrow \infty} \sum_{k=0}^{N-1}\left(\mathrm{E}\left(X_{1}^{2} \cdot g_{k}\right) \sum_{j=k+1}^{N} \frac{1}{j^{2}}\right) \\
& \leq \frac{\pi^{2}}{6} \lim _{N \rightarrow \infty} \sum_{k=0}^{N-1} \frac{\mathrm{E}\left(X_{1}^{2} \cdot g_{k}\right)}{k+1} \\
& =\frac{\pi^{2}}{6} \sum_{k=0}^{\infty} \frac{1}{k+1} \int_{\left\{k<X_{1} \leq k+1\right\}} X_{1}^{2} \mathrm{dP} \\
& \leq \frac{\pi^{2}}{6} \sum_{k=0}^{\infty} \frac{k+1}{k+1} \mathrm{E}\left(X_{1} \cdot g_{k}\right)=\frac{\pi^{2}}{6} \mathrm{E} X_{1}<\infty .
\end{aligned}
$$

It remains to show that $X_{n}-Y_{n} \rightarrow 0$ as $n \rightarrow \infty$ in $L^{1}$-sense and a.s. First, I will prove the $L^{1}$ convergence. We have $\mathrm{E}\left|X_{n}-Y_{n}\right|=\mathrm{E}\left(X_{n}-Y_{n}\right)=\mathrm{E}\left(X_{n} \cdot 1_{\left\{X_{n}>n\right\}}\right)=\mathrm{E}\left(X_{1} \cdot 1_{\left\{X_{1}>n\right\}}\right)$. Since $\mathrm{E} X_{1}<\infty$ by assumption, $\mathrm{E}\left(X_{1} \cdot 1_{\left\{X_{1}>n\right\}}\right)$ will converge to 0 , as $n \rightarrow \infty$, by Lebesgue's dominated convergence Theorem.

Now, regarding the a.s. convergence, we have

$$
\sum_{n=1}^{\infty} \mathrm{P}\left(X_{n} \neq Y_{n}\right)=\sum_{n=1}^{\infty} \mathrm{P}\left(X_{n}>n\right)=\sum_{n=1}^{\infty} \mathrm{P}\left(X_{1}>n\right) \leq \int_{0}^{\infty} \mathrm{P}\left(X_{1} \geq x\right) \mathrm{d} x=\mathrm{E} X_{1}<\infty .
$$

Hence, by Borel-Cantelli, with probability 1 it is true that $X_{n}=Y_{n}$ for $n$ large enough.

\section{Acknowledgements}

I would like to thank Jordan Stoyanov for his interest in my work and for his many useful comments and suggestions, most of which are taken into account in this version of the paper. 


\section{References}

[1] Nasrollah Etemadi, An elementary proof of the Strong Law of Large Numbers. Zeitschrift für Wahrscheinlichkeitstheorie und Verwandte Gebiete 55, pages 119-122 (1981). https://doi.org/10.1007/BF01013465

[2] Valery Korchevsky, On the Strong Law of Large Numbers for Sequences of Pairwise Independent Random Variables. J Math Sci 244, pages 805-810 (2020). https://doi.org/10.1007/s10958-020-04654-y

[3] Tapas K. Chandra and A. Goswami, Cesáro Uniform Integrability and the Strong Law of Large Numbers. Sankhyā: The Indian Journal of Statistics, Series A (1961-2002), vol. 54, no. 2, 1992, pages 215-231. https://www.jstor.org/stable/25050875

[4] Albert N. Shiryaev, Probability 2. Third english edition, 2018. Part of the Springer Graduate Texts in Mathematics book series (GTM, volume 95). https://doi.org/10.1007/978-0-387-72208-5

[5] SÁndor Csörgő and K. TAndori and Vilmos Totik, On the Strong Law of Large Numbers for pairwise independent random variables. Acta Math Hung 42, pages 319-330 (1983). https://doi.org/10.1007/BF01956779

[6] Jordan M. Stoyanov, Counterexamples in Probability. Third edition, Dover, Mineola (NY), 2014. Part of Dover Books on Mathematics. https://www.worldcat.org/title/counterexamples-in-probability/oclc/958218408 